\title{
La France, l'Allemagne et l'Ancien Testament. L'éternelle quête de
} l'identité

Thomas Romer

\section{Citer ce document / Cite this document :}

Romer Thomas. La France, l'Allemagne et l'Ancien Testament. L'éternelle quête de l'identité. In: Autres Temps. Cahiers d'éthique sociale et politique. $\mathrm{N}^{\circ} 65,2000$. pp. 18-23;

doi : https://doi.org/10.3406/chris.2000.2178

https://www.persee.fr/doc/chris_0753-2776_2000_num_65_1_2178

Fichier pdf généré le 26/03/2019 


\title{
La France, l'Allemagne et l'Ancien Testament, l'éternelle quête de l'identité
}

\author{
Thomas Römer *
}

\section{Deux identités}

Lorsque je suis arrivé en 1980 à Paris, je fus frappé par le nombre important de citoyens français qui ne ressemblaient pas au « Français moyen ». On pouvait être d'origine africaine, asiatique, maghrébine et néanmoins se comprendre comme français; alors que dans mon Allemagne natale, il existait extrêmement peu d'Allemands de couleur noire, jaune ou basanée.

L'explication de cette surprise (naïve sans doute) est apparemment simple. Alors que la France pratique un «droit du sol » et facilite la naturalisation des personnes ayant d'autres origines, mais étant nées en France, l'Allemagne pratique un "droit du sang » et reste profondément attachée à la généalogie. En Allemagne, il est très difficile pour des jeunes Turcs, nés sur le sol allemand, d'obtenir la nationalité allemande, tandis que de lointains descendants des paysans allemands émigrés en Russie au $\mathrm{XIX}^{\mathrm{e}}$ siècle la reçoivent quasi automatiquement, même s'ils ne parlent pas un mot d'allemand. Il leur suffit de pouvoir attester un arrière grand-père d'origine germanique.

L'identité française, quant à elle, peut être comprise comme une «identité vocationnelle ». On est (ou devient) français du moment que l'on souscrit aux grands principes de la République française «liberté, égalité, fraternité ». Cette identité se construit autour d'une souveraineté forte et centralisée. C'est le Président de la République, à plusieurs égards héritier de l'idéologie royale, qui fonctionne comme médiateur entre les principes de la République et le «peuple français ». Ce rôle explique l'ambiance hautement émotionnelle qui règne en France lors des élections présidentielles où le peuple est invité à choisir celui qui représente le mieux l'identité française vis-à-vis de l'intérieur et vis-à-vis de

\footnotetext{
* Professeur d'Ancien Testament à la faculté de Théologie de l'Université de Lausanne, dont il est actuellement le doyen, Thomas Römer est allemand et a fait une grande partie de ses études en France.
} 
La France, l'Allemagne et l'Ancien Testament, l'éternelle quête de l'identité

l'extérieur. L'identité centralisée s'exprime également par le statut tout particulier de Paris. Tout salut vient de la capitale. C'est là que se trouvent toutes les institutions, c'est là qu'on décide de l'éducation pour le pays entier, c'est là que se fait la France. N'importe quel Français est censé connaître Paris, même s'il n'y a jamais mis les pieds.

Rien de tel en Allemagne. Ni le Président ni le chancelier jouent dans la construction de l'identité allemande un rôle comparable au Président français. Et même si la nouvelle capitale de l'Allemagne est une mégalopole, Berlin ne sera jamais Paris. L'identité des Allemands est d'abord régionale et fédérale, elle est d'une certaine manière généalogique.

Au moment de l'existence des deux états allemands, les habitants de la République fédérale utilisaient, presque comme une incantation, l'expression « nos frères à l'Est » pour conjurer l'unité allemande.

Cette opposition entre l'identité vocationnelle française et l'identité généalogique allemande est bien entendu caricaturale, mais elle permet tout de même de mettre en relief les deux modèles différents qui peuvent être présents dans la construction de l'identité d'un groupe. Ces deux modèles sont en fait déjà attestés dans l'écrit fondateur de la tradition judéo-chrétienne, à savoir la Bible hébraïque.

\section{Identité vocationnelle et identité généalogique}

Dans le livre d'Osée on trouve un poème étonnant qui oppose l'ancêtre Jacob à Moïse et l'exode :

Os 12. 13-14: «Jacob s'enfuit aux plaines d'Aram et Israël servit pour une femme, et, pour une femme il se fit gardien de troupeaux. Mais, par un prophète, le SEIGNEUR a fait monter Israël hors d'Égypte, et par un prophète, Israël a été gardé ».

Os 12 contient une récapitulation extrêmement négative de l'histoire de l'ancêtre Jacob, qui a été fraudeur et tricheur dès sa naissance. Si l'on compare ce texte avec les récits sur Jacob dans la Genèse, on se rend vite compte que les exploits de Jacob célébrés en Gn 25-36 sont critiqués et condamnés en Os 12. Mais, ce dernier texte ne s'arrête pas à la simple critique. A la référence à Jacob, il oppose la médiation prophétique et le fait que le « vrai » Dieu d'Israël n'est pas le Dieu de Jacob, mais celui qui fait sortir d'Égypte. Les jeux de mots à la fin du poème sont particulièrement révélateurs: Jacob/Israël se fait esclave à cause d'une femme, et à cause d'une femme, il devient gardien de troupeau. Dieu, par contre, a libéré son peuple de l'esclavage égyptien et l'a gardé par un prophète, c'est-à-dire Moïse. La référence à la femme n'est pas seulement une 
allusion à la faiblesse de Jacob qui fait tout et n'importe quoi à cause de son attirance par «le sexe faible », l'enjeu est plus profond. Le renvoi à l'histoire des épouses de Jacob vise ici le modèle identitaire qui passe par la descendance, donc par la généalogie. Ce qui fait Israël pour l'auteur d'Os 12 ce n'est pas la «femme », c'est-à-dire l'appartenance à un système généalogique, mais c'est la parole de Yahvé, transmise et acceptée par une médiation prophético-mosaïque. Ce modèle est développé dans le livre du Deutéronome et ensuite dans les livres historiques influencés par le style et la théologie deutéronomistes.

Le livre du Deutéronome appelle ses destinataires «tout Israël » sans trop se préoccuper des différenciations tribales et claniques. On ne trouve aucune liste généalogique dans le Deutéronome. Ce qui importe, c'est d'entrer dans l'alliance de Dieu, c'est-à-dire accepter un projet de société dont la Tora (la loi deutéronomique en Dt 12ss) est le médiateur. Fait partie d'Israël celui qui observe et met en pratique les règles de société définies dans le Dt.

\section{Le conflit des identités}

Le Deutéronome, dans sa forme actuelle, présuppose la situation historique de l'exil et joue sur l'équivalence entre la situation du peuple au désert et celle des exilés. C'est dans ce contexte-là qu'il faut comprendre les mentions très fréquentes des « pères » dans le Deutéronome et dans les livres historiques. Dans le cadre de l'historiographie deutéronomiste, ces pères sont des « anti-ancêtres ». Ils ne désignent nullement les Patriarches Abraham, Isaac et Jacob, comme l'on l'a pensé souvent, mais ils renvoient à l'Égypte, à l'exode et à la conquête du pays. L'identification des pères aux patriarches ( $p$. ex. en Dt 1,8 et 6,10 ) est l'œuvre des derniers rédacteurs qui ont fait du Deutéronome le dernier livre du Pentateuque, cherchant alors à créer à l'intérieur du Deutéronome des renvois à la Genèse.

En ce qui concerne les pères «originels » du Deutéronome, aucune généalogie n'est proposée qui permettrait aux destinataires de trouver un lien avec ces pères par le sang. Les pères deutéronomistes évoquent l'Égypte et permettent ainsi aux exilés de tracer un parallèle entre leur situation et la situation en Égypte. En s'adressant à une partie des exilés, les Deutéronomistes leur proposent un mythe d'origine exodique.

La population non-exilée, par contre, essaie de reconstruire son identité autour de la figure d'Abraham. Ceci est attesté par un passage du livre d'Ezéchiel (Ez 33.24) : «Fils d'homme, les habitants de ces ruines qui se trouvent sur le sol d'Israël disent : «Abraham qui était seul a possédé le 
pays ; nous qui sommes nombreux, c'est à nous que le pays est donné en possession ».

Selon ce texte, la population autochtone justifie son droit à la possession du pays contre les revendications des déportés (cf. également Ez 11,15) en ayant recours, via la figure d'Abraham, à un mélange de droit du sol et de droit du sang. Abraham est invoqué à la fois comme ancêtre mais également comme habitant depuis toujours le pays (cf. aussi Es 51,2 où Abraham est comparé à une sorte de rocher primordial). L'auteur d'Ez 33,24ss, qui, comme les auteurs de l'historiographie deutéronomiste, représente les intérêts des exilés à Babylone, condamne, dans la suite de l'oracle, violemment cette revendication.

La contestation des revendications territoriales et identitaires de la population non-exilée par les représentants des déportés fait apparaître une sorte de contradiction chez les détenteurs du modèle généalogique. Ils s'adressent théoriquement à tout Israël mais en réalité le « tout Israël » est limité aux Juifs exilés à Babylone. Cette tension entre un discours, a priori ouvert, et des prétentions partisanes s'est perpétuée à travers les siècles.

\section{Des cartes brouillées}

Chez les tenants de l'identité vocationnelle pour lesquels l'identité d'Israël face à son Dieu se définit dans l'adhésion au projet de Dieu (l'alliance), on trouve cependant des textes d'une fermeture inquiétante à l'égard des autres. Ainsi, Dt 7,1-6 interdit tout contact entre « Israël » et les autres, prônant même des actes de violence pour arriver à la séparation totale. Or, à y regarder de près, les « autres nations » en Dt 7 font historiquement et sociologiquement partie d'Israël. En Dt 7,2 figure une liste stéréotypée des peuples dont Israël doit se séparer (Hittites, Amorites, Cananéens, ...). On trouve une énumération de peuples comparable à celle de Dt 7 dans le chapitre 9 du livre d'Esdras. Comme en Dt 7, il y est question de l'interdiction des «mariages mixtes». Or, il s'agit en réalité de l'interdiction des mariages entre les (anciens) exilés et la population restée dans le pays. C'est cette dernière qui est identifiée en Esd 9,12 aux peuples étrangers. C'est une tentative d'exclure d'Israël une partie de la population qui sociologiquement en fait partie. Du coup, l'identité vocationnelle deutéronomiste court le risque de se pervertir en un «droit du sang » qui a un goût d'inceste.

De l'autre côté, le modèle généalogique qu'on aurait pu suspecter de véhiculer un modèle identitaire fermé se transforme, avec la mise par écrit de l'histoire d'Abraham, pendant et après l'exil, en un modèle 
d'ouverture exemplaire. Abraham est certes devenu ' l'ancêtre d'Israël via Isaac et Jacob, mais il est en même temps l'ancêtre des Arabes via Ismaël et des Moabites et Ammonites (les Jordaniens d'aujourd'hui) via Lot. Certes, via Abraham, Israël dit son droit au pays, mais ce droit n'est jamais accompagné dans la Genèse par l'expulsion des autres. D'ailleurs, quand Dieu annonce à Abraham la possession du pays pour sa descendance, il n'est jamais dit qu' Ismaël serait exclu de cette descendance.

D'une manière plus générale, le livre de la Genèse construit un grand système généalogique qui fait de quasiment tous les peuples de la terre une famille. Le droit du sang est subverti.

\section{Attention aux dérives}

Les éditeurs de la Bible hébraïque n'ont pas tranché entre le modèle vocationnel et le modèle généalogique. Ils les ont plutôt fait cohabiter, même s'il s'agit souvent d'une cohabitation conflictuelle. Le Pentateuque s'ouvre avec le modèle généalogique $(\mathrm{Gn})$ et se termine avec le modèle vocationnel (Dt). Il faut essayer de penser les deux concepts ensemble. Comme illustration de cette nécessité, les derniers rédacteurs du Pentateuque ont identifié les pères du Dt aux Patriarches de la Genèse (cf. surtout Dt 1,8 et 34,4). Les deux modèles d'identité possèdent leurs chances et leurs dangers. Le modèle vocationnel qui pourrait partiellement être assimilé à un droit du sol permet en théorie d'intégrer tous ceux qui souscrivent à un projet commun de société ; mais, le danger existe que ce projet devienne tout à coup un concept autoritaire et fermé. De l'autre côté, le modèle généalogique, sorte de droit du sang, apparaît a priori potentiellement raciste. Mais tout dépend de comment on comprend les « liens du sang ». En insistant sur les liens qui unissent les habitants d'un même pays, d'un même continent, de la terre entière, l'idée généalogique peut aussi devenir facteur d'intégration tout en respectant les différences entre les uns et les autres.

Peut-on actualiser ces constats vétérotestamentaires ? Il serait contraire à la tradition biblique et réformée de donner l'illusion d'une immédiateté du texte biblique. On peut néanmoins essayer de confronter l'enquête sur l'identité dans l'Ancien Testament aux situations de la France et de l'Allemagne.

\section{Identité et convivialité}

A priori, le modèle français paraît plus ouvert. Mais une identité nationale qui se construit en grande partie autour du concept de la 
La France, l'Allemagne et l'Ancien Testament, l'éternelle quête de l'identité

souveraineté court le risque de devenir une identité qui a des difficultés à assumer la diversité. Le problème des banlieues est à cet égard éloquent. Peut-on être français et musulman ? Le psychodrame du foulard a fait apparaître des conflits de loyauté qui peuvent surgir à l'intérieur d'un modèle identitaire institutionnel.

De nombreux politiciens et journalistes français fantasment sur la montée de l'extrême droite en Allemagne, alors que c'est en France que le Front national récolte depuis une vingtaine d'années lors des élections entre dix et vingt pour-cent des voix. L'Allemagne (qu'il faut alors bien distinguer de l'Autriche) ne connaît pas de parti extrémiste d'une importance comparable. L'idéologie généalogique entretenue par Le Pen et Mégret («La France aux Français ») peut aussi se lire comme réaction contre un État perçu comme trop «institutionnalisé ».

Il n'est nullement mon intention de défendre dans ces lignes le droit du sang à l'allemande. Mais, la construction identitaire allemande contient peut-être, notamment par l'importance du fédéralisme, des potentialités de construire une identité dans la diversité. Prenons un exemple anecdotique mais révélateur: lors du concours Eurovision de la chanson en 1999, l'Allemagne s'est fait représenter par une chanson qui, en grande partie était interprétée en langue turque. Il est très difficile d'imaginer que la France puisse sélectionner des chansons dans d'autre langues que le français.

Une identité liée à une forte souveraineté fait sans doute peu de place à la généalogie ; cependant, elle a parfois du mal à intégrer des minorités qui veulent garder leur culture et leurs racines spécifiques.

Une identité généalogique peut certes tomber dans des dérives racistes, mais elle peut aussi ouvrir la généalogie vers une convivialité et construire avec les autres une «grande famille» (cf. l'exemple d'Abraham).

Ainsi sommes-nous amenés à abandonner quelques idées trop simplistes dans le débat entre droit du sol et droit du sang. Seul, celui qui sait qui il est, peut accueillir l'autre. Mais, celui qui pense que son identité se définit par une formule creuse (du style «La France aux Français») restera toujours enfermé sur lui-même. Ce n'est pas par hasard si Jean Baptiste, dans le Nouveau Testament, reprend la critique prophétique en s'adressant à ceux qui savent trop bien qui ils sont : « Ne vous avisez pas de dire en vous-mêmes : «nous avons pour père Abraham ». Car je vous le dis, des pierres que voici, Dieu peut susciter des enfants à Abraham » (Mt 3,9). 